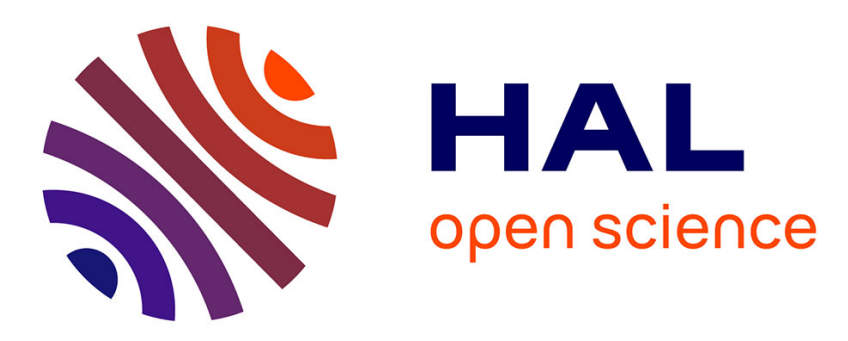

\title{
Cubosomal lipid nanoassemblies with pH-sensitive shells created by biopolymer complexes: A synchrotron SAXS study
}

Patrick D Mathews, Omar Mertins, Borislav Angelov, Angelina Angelova

\section{- To cite this version:}

Patrick D Mathews, Omar Mertins, Borislav Angelov, Angelina Angelova. Cubosomal lipid nanoassemblies with pH-sensitive shells created by biopolymer complexes: A synchrotron SAXS study. Journal of Colloid and Interface Science, 2022, 607, pp.440-450. 10.1016/j.jcis.2021.08.187 . hal03445424

\author{
HAL Id: hal-03445424 \\ https://hal.science/hal-03445424
}

Submitted on 23 Nov 2021

HAL is a multi-disciplinary open access archive for the deposit and dissemination of scientific research documents, whether they are published or not. The documents may come from teaching and research institutions in France or abroad, or from public or private research centers.
L'archive ouverte pluridisciplinaire HAL, est destinée au dépôt et à la diffusion de documents scientifiques de niveau recherche, publiés ou non, émanant des établissements d'enseignement et de recherche français ou étrangers, des laboratoires publics ou privés. 


\title{
Cubosomal lipid nanoassemblies with pH-sensitive shells created by biopolymer complexes: A synchrotron SAXS study
}

\author{
Patrick D. Mathews ${ }^{a}$, Omar Mertins ${ }^{\mathrm{a}, \mathrm{b},{ }^{*}}$, Borislav Angelov ${ }^{\mathrm{c}}$, Angelina Angelova ${ }^{\mathrm{b},{ }^{*}}$ \\ a Laboratory of Nano Bio Materials (LNBM), Department of Biophysics, Paulista Medical \\ School, Federal University of Sao Paulo (UNIFESP), 04023-062 Sao Paulo, Brazil \\ ${ }^{b}$ Institut Galien Paris-Saclay UMR8612, Université Paris-Saclay, CNRS, F-92296 Châtenay- \\ Malabry, France \\ 'Institute of Physics, ELI Beamlines, Academy of Sciences of the Czech Republic, CZ-18221 \\ Prague, Czech Republic \\ *Corresponding authors: \\ mertins@unifesp.br (O. Mertins); angelina.angelova@universite-paris-saclay.fr (A. Angelova)
}

\section{Abstract}

We report a strategy for sustainable development of $\mathrm{pH}$-responsive cubic liquid crystalline nanoparticles (cubosomes), in which the structure-defining lyotropic nonlamellar lipid and the eventually encapsulated guest molecules can be protected by $\mathrm{pH}$-sensitive polyelectrolyte shells with mucoadhesive properties. Bulk non-lamellar phases as well as $\mathrm{pH}-$ responsive polyelectrolyte-modified nanocarriers were formed by spontaneous assembly of the nonlamellar lipid monoolein and two biopolymers tailored in nanocomplexes with $\mathrm{pH}-$ dependent net charge. The mesophase particles involved positively charged $N$-argininemodified chitosan (CHarg) and negatively charged alginate (ALG) chains assembled at different biopolymer concentrations and charge ratios into a series of $\mathrm{pH}$-responsive complexes. The roles of Pluronic F127 as a dispersing agent and a stabilizer of the nanoscale dispersions were examined. Synchrotron small-angle X-ray scattering (SAXS) investigations were performed at several $\mathrm{N}$-arginine-modified chitosan/alginate ratios (CHarg/ALG with 10, 15 and 20 wt\% ALG relative to $\mathrm{CHarg}$ ) and varying $\mathrm{pH}$ values mimicking the $\mathrm{pH}$ conditions of the gastrointestinal route. The structural parameters characterizing the inner cubic liquid crystalline organizations of the nanocarriers were determined as well as the particle sizes and stability on storage. The surface charge variations, influencing the measured zetapotentials, evidenced the inclusion of the CHarg/ALG biopolymer complexes into the lipid nanoassemblies. The polyelectrolyte shells rendered the hybrid cubosome nanocarriers $\mathrm{pH}$ sensitive and influenced the swelling of their lipid-phase core as revealed by the acquired SAXS patterns. The pH-responsiveness and the mucoadhesive features of the cubosomal lipid/polyelectrolyte nanocomplexes may be of interest for in vivo drug delivery applications. 
Keywords: self-assembled lipid/biopolymer complexes, cubic phase nanoparticles (cubosomes), N-arginine-modified chitosan; hybrid nonlamellar liquid crystalline nanostructures; synchrotron small-angle X-ray scattering (SAXS); drug delivery systems for oral administration.

\section{Introduction}

Lipid-based cubic liquid crystalline particles (cubosomes) represent nanostructured colloidal delivery systems with enhanced capacity for encapsulation of biomolecules, drugs, and other bioactive ingredients [1-4]. They have found applications in the development of various controlled release carriers and nanoformulations [5-7]. The inner liquid crystalline organization, comprising periodic lipid membranes and networks of aqueous channels, is advantageous for the entrapment, solubilization and protection of single or multiple therapeutic compounds [5-10,11]. The innovative aspects of the cubosomes topology relay in the inner structure of these nanocarriers, which consists of liquid crystalline lipid bilayers organized in defined cubic lattice architectures [12]. Cubosomes derived from bicontinuous lipid cubic phases and involving two labyrinthine three-dimensional (3D) networks of aqueous channels have a significantly larger surface area than the liposome membranes [13]. At same particle sizes as those of the vesicular carriers, the 3D cubosome structure is capable of encapsulating larger amounts of hydrophobic and hydrophilic substances as compared to liposomes. Therefore, the principal advantage of cubosomes over liposomes lies in the liquid crystalline organization, which can provide sustained drug release over prolonged times with regards to liposomes in contact with cellular interfaces [14].

$\mathrm{pH}$-responsive cubosome, hexosome, and spongosome liquid crystalline lipid nanoparticles are of strong current interest for biomedical applications [10,15-17]. Such carriers can be prepared by dispersion of mixed nonlamellar lipid phases in the presence of surfactant agents facilitating the bulk phase fragmentation as well as the steric stabilization of the created nanosized particles $[1,2,8,18-28]$. Cubosome particles have been functionalized by various kinds of polymer shells in order to improve their surface properties and stability [2228]. Depending on the investigated environment, the conventionally used Pluronic copolymer surfactants have been replaced by polymers of different compositions and topologies (e.g. PEGylated amphiphiles, liner or branched polyethylenimine (PEI), dendrimers, polyelectrolytes, etc.). Some of the created hierarchical lipid/polymer assemblies have been biocompatible and have enabled the fabrication of sophisticated drug delivery systems with stimuli-responsive properties $[22,23,27]$.

The polysaccharide chitosan has attracted strong attention in the development of nonlamellar lyotropic liquid crystalline nanoparticles with tailored polyelectrolyte functionalities [29-33]. Chitosan-modified cubosome lipid particles have been elaborated by inspiration 
from liposomes, which were modified by anchored polyelectrolytes or functional macromolecules [34-44]. In fact, liposomes formed by charged lipids and polyelectrolytes represent colloid delivery systems with high affinity for the mucosal and the cellular membranes [37-39]. Liposomes with significant mucoadhesive features have been used as delivery systems in the treatment of various types of cancers [34-36]. Mucoadhesion favors the absorption of the active substances, transported by the liposome carriers, and leads to increased drug bioavailability [40-42]. Liposomes associated with chitosan have been exploited in the development of nanomaterials for encapsulation of drugs, plasmid DNA and vaccines [43-46]. It has been shown that the coating of liposomes by chitosan leads to significant mucoadhesion of the nanostructures as well as to increased bioavailability of the encapsulated active agents [47-49].

Chitosan is a polymer of natural origin, biodegradable, non-toxic, non-antigenic, biocompatible, and displaying antimicrobial activities. These properties make chitosan a biopolymer of wide applicability in colloidal systems as it contributes to the improvement of their characteristics such as stability, retention of encapsulated material, and specificity for applications where bioadhesion is required. The interaction of chitosan with the lipid bilayers in liposome systems or giant vesicles has been well documented [50-53]. In order to further enhance the functionality of the polysaccharide chitosan, chemical anchoring of active ingredients such as arginine has been performed [44,54]. The $\mathrm{N}$-arginine-modified chitosan derivative has been advantageous in the development of new generation of liposomes with specific functional characteristics and mucoadhesive properties $[44,54]$.

A recent SAXS study has shown that lipids associated with the polysaccharide chitosan can assemble into certain cubic phases depending on the lipid/polymer mass ratio and the degree of hydration [55]. Moreover, the association of chitosan and chitosan- $N$-arginine with alginate, another polysaccharide with advantageous characteristics, allowed the production of $\mathrm{pH}$-sensitive nanoparticles as drug delivery systems for the anthelmintic drugs ivermectin, mebendazole and praziquantel [56-59]. Indeed, chitosan and alginate are oppositely charged polyelectrolytes in aqueous medium, which renders the association of both macromolecules $\mathrm{pH}$-dependent [54]. In vivo studies of these nanoparticles in fish animals infected with gastrointestinal parasites have demonstrated the excellent treatment efficacy upon oral administration [54,58,59]. In parallel, the performed physicochemical studies have suggested that the structural changes of the chitosan/alginate nanoparticles, as a function of $\mathrm{pH}$, play a crucial role for the observed in vivo effects [54,58].

In a further step of the upgrade of the hierarchical core-shell topologies and the surface properties of the cubosomal nanoarchitectures, we report here nonlamellar lipid nanoparticles with $\mathrm{pH}$-responsive biopolymer shells. The latter are created as polyelectrolyte complexes of oppositely charged chitosan- $N$-arginine (CHarg) and alginate (ALG) derivatives. The feasibility of production of biopolymer-tailored lipid cubosomes is evidenced by the 
liquid crystalline organizations, which were determined by synchrotron SAXS for various lipid/polysaccharide compositions at several $\mathrm{N}$-arginine-modified chitosan/alginate (CHarg/ALG) ratios. The stability and the $\mathrm{pH}$-responsiveness of the hybrid cubosomal nanoparticle structures with tailored polysaccharide ( $\mathrm{N}$-arginine-chitosan/alginate) complexes were examined under $\mathrm{pH}$ conditions mimicking the $\mathrm{pH}$ values in the gastrointestinal route. As a matter of fact, the oral administration of drugs is known to be more convenient and accessible. Due to the hydrophobic nature of several bioactive substances and their low solubility, this route of drug administration is still challenging [60]. With the development of lipid nanoparticle carriers such as cubosomes (among other types), the oral route may become a reality in the treatment of cancer $[17,61,62]$. Furthermore, the controlled release of drugs from $\mathrm{pH}$-sensitive cubosomes into the gastrointestinal tract may lead to various pharmacological advantages $[17,61,63]$.

In this work, the structural characteristics of self-assembled lipid nanoparticles functionalized with chitosan- $N$-arginine ( $\mathrm{CHarg}$ ) and alginate (ALG), associated in polyelectrolyte complexes, are studied in detail by synchrotron SAXS, dynamic light scattering and zeta potential measurements. The structural variations of the lipid nanocarriers associated with different ratios of the two polysaccharides are expected to render the cubosome structural parameters responsive to $\mathrm{pH}$ of the aqueous medium. Thus, the obtained hybrid colloidal systems, comprised by lipidic cubosomes with $\mathrm{pH}$-responsive chitosan- $N$-arginine ( $\mathrm{CHarg}$ )/alginate (ALG) shells may be promising for applications that involve oral drug administration.

\section{Materials and methods}

\subsection{Materials}

The lipid monoolein (99\%) and Pluronic F127 (99\%) both in powder form (Sigma-Aldrich) were dissolved in chloroform at 200 and $100 \mathrm{mg} / \mathrm{mL}$, respectively, and kept at $-20{ }^{\circ} \mathrm{C}$ until use in mixed systems at desired ratios. Chitosan- $\mathrm{N}$-arginine ( $\mathrm{CHarg}$ ) was prepared, purified and characterized as previously described [54] with degree of deacetylation of 95\%, average molecular weight $\left(\mathrm{M}_{\mathrm{w}}\right)$ of $135 \mathrm{kDa}$, and a yield of $3.5 \%$ of monomers anchored to arginine. Alginate (ALG) was from Sigma-Aldrich with $\mathrm{M}_{\mathrm{w}}=200 \mathrm{kDa}$ and containing $61 \%$ mannuronic acid and $39 \%$ guluronic acid units. The polysaccharides were dissolved in water at $5 \mathrm{mg} / \mathrm{mL}$ with overnight stirring followed by 5 min bath sonication. Buffers with concentration $0.2 \mathrm{M}$ were prepared with sodium acetate and acetic acid to obtain solutions with $\mathrm{pH} 2.0$ and 5.0.

Disodium phosphate and citric acid were used to prepare buffers with $\mathrm{pH} 7.4$ and 8.0 by changing the proportion between the components at same total concentration of $0.2 \mathrm{M}$. All reagents were of analytical grade. Water was purified from MilliQ system (Millipore Corp.) to a total organic carbon value of less than $15 \mathrm{ppb}$ and resistivity of $18 \mathrm{M} \Omega . \mathrm{cm}$.

\subsection{Bulk liquid crystalline phase preparation}


Bulk cubic mesophases were prepared with $20 \mathrm{mg}$ of pure monoolein (MO) and $80 \mathrm{mg}$ of aqueous buffer solutions or pure water. The solvent chloroform was evaporated from the lipid phase under nitrogen gas stream in individual glass vials and the obtained monoolein lipid films were lyophilized under cooling and reduced pressure overnight. Bulk liquid crystalline samples were produced by adding a volume of hydration water or buffers of varying $\mathrm{pH}$ values, followed by homogenization through 2 min vortex mixing and 5 min bath sonication. Ice was included in the sonicator water bath to avoid heating of the samples. The samples were sealed and kept at $4{ }^{\circ} \mathrm{C}$ until SAXS measurements.

\subsection{Cubosome nanoparticle preparation}

Diverse cubosome dispersions were prepared using Pluronic F127 (PF127), as a dispersing agent and stabilizer, and chitosan- $N$-arginine (CHarg) and alginate (ALG) as a polyelectrolyte system, which can form charged biopolymer complexes with affinity for the intestinal mucosal membrane. All samples were equally prepared with $20 \mathrm{mg}$ of monoolein (MO) yielding a stock concentration of $135 \mathrm{mM}$ for the lipid. Both MO lipid and PF127 were mixed in chloroform in glass vials and the solvent was evaporated as described above. Samples were prepared with 7, 9, 11, 13 and $15 \mathrm{wt} \%$ of PF127 relative to MO. After lyophilization of the mixed films, pure water was added and the samples were submitted 10 times to a vortex-sonication cycles comprising 1 min vortexing and 5 min sonication. For the $7 \mathrm{wt} \%$ Pluronic F127 content, additional samples were prepared in the same manner but with

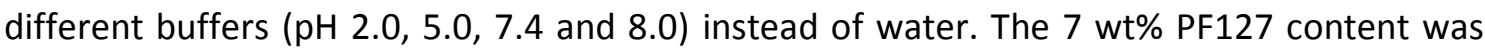
chosen for the production of biopolymer-modified cubosomes based on the experimental

procedure illustrated in Scheme 1. The CHarg and ALG solutions were added in a series of different amounts and relative proportions to the vials containing the dried lipid films. This led to the production of systems involving 10, 15 and $22 \mu \mathrm{M}$ of polysaccharides. Every one included 10, 15 or $20 \mathrm{wt} \%$ ALG proportion relative to CHarg. All sample volumes were adjusted to $264 \mu \mathrm{L}$ by adding pure water when required. The vials were sealed and subjected to cycles of 10 times vortexing-sonication in ice bath and then kept for equilibration in the dark at room temperature $\left(22-25^{\circ} \mathrm{C}\right)$ for one week. Subsequently, $150 \mu \mathrm{L}$ of each of the four different $\mathrm{pH}$ buffers was added to every vial of each biopolymer concentration and each relative proportion of the polysaccharides. The employed procedure led to buffer dilution to $72.5 \mathrm{mM}$ concentration. Totally, $36 \mathrm{lipid} /$ biopolymer nanoparticle samples were produced for varying $\mathrm{pH}$ and $\mathrm{CHarg} / \mathrm{ALG}$ ratios with a final volume of $414 \mu \mathrm{L}$ each. The samples were further subjected to $1 \mathrm{~min}$ vortexing and $5 \mathrm{~min}$ bath sonication and then kept at rest for one more week before structural analysis. 


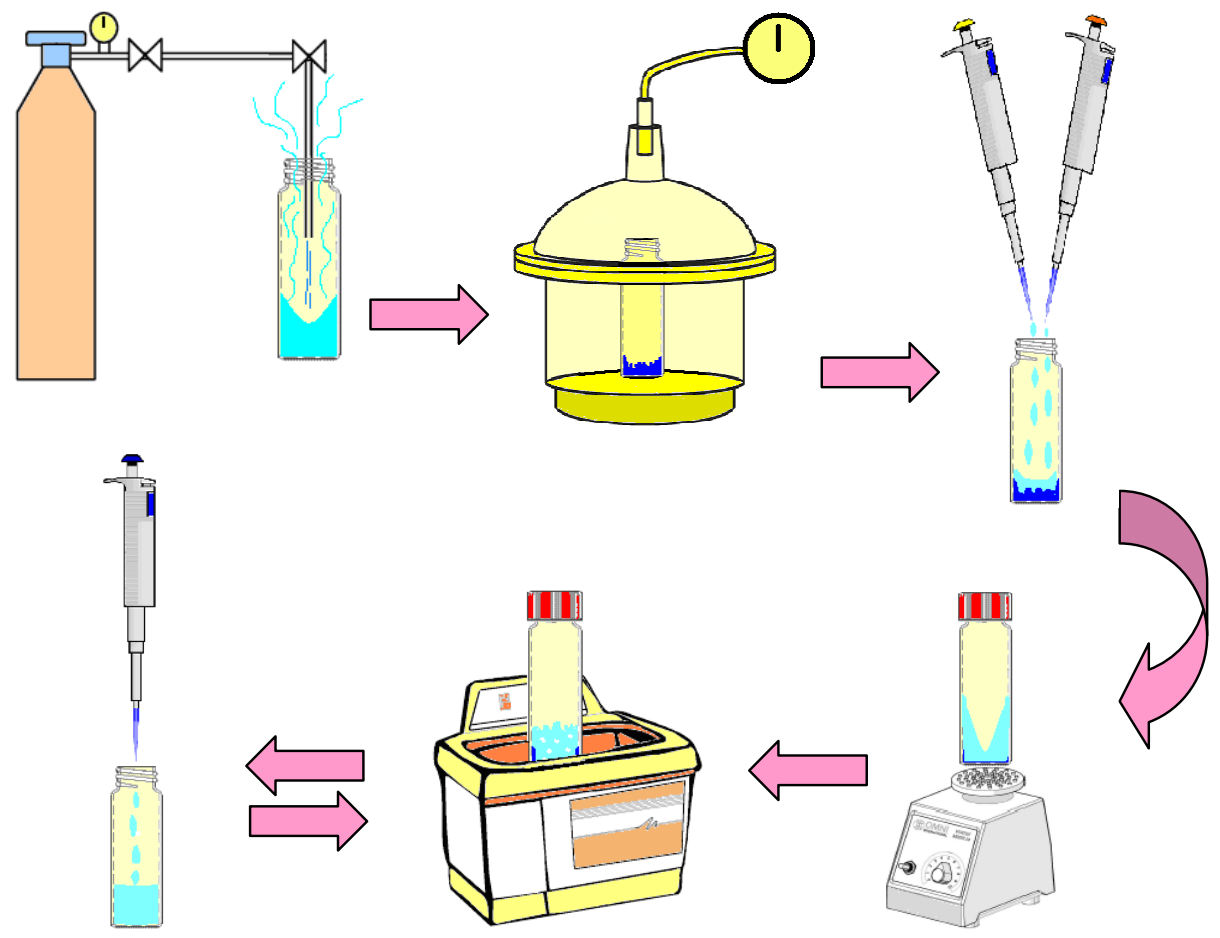

Scheme 1. Preparation protocol of lipid/biopolymer tailored cubosomes by self-assembly. (i) After mixing of the ingredients, the solvent chloroform is evaporated from the vial containing the monoolein/Pluronic F127 mixture under nitrogen gas stream. (ii) The obtained thin film in the vial is lyophilized under cooling and reduced pressure overnight. (iii) The thin film is hydrated with solutions of chitosan- $N$-arginine (CHarg) and alginate (ALG) biopolymers. (iv) The mixture is subjected 10 times to cycles of $1 \mathrm{~min}$ vortex mixing and 5 min bath sonication in the presence of ice around the vials. (v) After one week equilibration, specific buffers are added and the sample is vortexed and sonicated again to obtain a fine dispersion of nanoparticles.

\subsection{Small angle $X$-ray scattering (SAXS)}

SAXS experiments were performed at the SWING beamline [64] of Synchrotron SOLEIL (Saint Aubin, France). The sample-to-detector distance was $3 \mathrm{~m}$. The patterns were recorded with a two-dimensional EigerX 4-M detector. The $q$-vector was defined as $q=(4 \pi / \lambda) \sin \theta$, where $2 \theta$ is the scattering angle. The synchrotron radiation wavelength was $\lambda=1.033 \AA$. The $q$-range calibration was done using a standard sample of silver behenate ( $d=58.38 \AA$ ). The investigated bulk phases and nanoparticle dispersions were filled in capillaries with a diameter of $1.5 \mathrm{~mm}$ and were sealed using paraffin wax. They were oriented in front of the $X$-ray beam $\left(25 \times 375 \mu \mathrm{m}^{2}\right)$ using a designed holder for multiple capillaries positioning $(x, y$, z). Exposure times of either $250 \mathrm{~ms}$ or $500 \mathrm{~ms}$ (for bulk lipid samples) or $1 \mathrm{~s}$ (for diluted dispersions) were used. No radiation damage was observed at these exposure times. The 
temperature was $22{ }^{\circ} \mathrm{C}$. Scattering patterns of an empty capillary, a capillary filled with MilliQ water and four capillaries for each buffer solution were recorded for intensity background subtractions. Data processing of the recorded 2D images was performed by the FOXTROT software [64]. An average of three spectra per capillary was acquired at two positions along the capillary length.

The lattice parameters $(a)$ of the liquid crystalline phases were derived from the Bragg peaks detected in the X-ray diffraction patterns. The assigned reflections were fitted through the Miller indexes according to the relation:

$$
q=(2 \pi / a)\left(\mathrm{h}^{2}+\mathrm{k}^{2}+\mathrm{I}^{2}\right)^{1 / 2}
$$

from linear fits of the plots of $q$ versus $\left(h^{2}+k^{2}+l^{2}\right)^{1 / 2}$ (see Figure S1), where $q$ is the peak position along the scattering vector axis and $h, k$, and $I$ are the Miller indices of the respective cubic lattice. The slope of the linear fit to the data equals the inverse of the cubic lattice parameter [65].

\subsection{Quasi-elastic light scattering and zeta potential}

Quasi-elastic light scattering (QELS) and zeta potential measurements were performed with a Nano-ZS90 Malvern ZetaSizer equipment (Malvern Instruments, UK) operating with a 4 $\mathrm{mW}$ HeNe laser at a wavelength of $632.8 \mathrm{~nm}$ in a temperature-controlled chamber at $25{ }^{\circ} \mathrm{C}$. Aliquots of $50 \mu \mathrm{L}$ of the stock cubosome dispersions were diluted with $1 \mathrm{~mL}$ of pure water or the corresponding buffer, in which the individual sample was prepared. QELS measurements were made with the samples in cuvettes and the scattering was acquired at an angle of $90^{\circ}$. The typical autocorrelation function was obtained using exponential spacing of the correlation time. The data analyses were performed with software provided by Malvern. The intensity-weighted size distribution was obtained by fitting data with a discrete Laplace inversion routine. Size determination was made using a Stokes-Einstein relation and the polydispersity was accessed by using cumulant analysis of the correlation functions measured by QELS applying the amplitude of the correlation function and the relaxation frequency. The second-order cumulant was used to compute the polydispersity of samples. Each analysis was a result of three consecutive measurements.

Zeta potential was concomitantly measured for the same samples by transferring the dispersions to the folded capillary zeta cell using a micropipette and changing the detection angle to $173^{\circ}$. Data was acquired performing at least 50 runs per sample. The principle of the measurement is based on laser Doppler velocimetry. The electrophoretic mobility was converted to zeta potential using the Helmholtz Smoluchowski relationship. The colloidal size was expressed as a hydrodynamic diameter in $\mathrm{nm}$ and zeta potential in $\mathrm{mV}$. 


\section{Results and discussion}

Hybrid lipid/biopolymer samples were prepared at varying $\mathrm{pH}$ and a number of chitosan- $\mathrm{N}$ arginine ( $\mathrm{CHarg}$ )/alginate( $\mathrm{ALG}$ ) ratios in the mixed compositions in order to unveil the structural characteristics of novel biopolymer-tailored cubosomes, which aim at development of $\mathrm{pH}$-responsive nanoparticles as vehicles for oral drug delivery applications.

\subsection{Determination of the concentration of the amphiphilic steric stabilizer favoring stable cubosomal dispersions}

For the described sample preparation method, we first determined the content of Pluronic F127, which favored mainly cubosomal structural organization of the lipid dispersions, and not other types of liquid crystalline nanoparticles (e.g. spongosomes, hexosomes, cubosomal precursors and intermediate structures). The sonication-vortex cycles, employed for bulk liquid crystalline phase fragmentation and dispersion, were expected to produce a coexistence of cubosomes and small vesicular membrane particles, which can merge into cubosomes on storage.

Figure 1A shows the SAXS profiles for the pure monoolein (MO) with different proportions of Pluronic F127 (PF127) used for lipid particle dispersion in pure water. The structural data for the 7 wt\% PF127 content evidenced a cubosomal $1 \mathrm{~m} 3 \mathrm{~m}$ liquid crystalline structure. It is confirmed by the indexing of the detected Bragg peaks for a cubic lattice, for which the peak positions are spaced in the ratios $\sqrt{ } 2: \sqrt{ } 4: \sqrt{ } 6: \sqrt{ } 10: \sqrt{ } 12: \sqrt{ } 14$. The lattice parameter of the inner $1 \mathrm{~m} 3 \mathrm{~m}$ cubosome structure was estimated to be $14.0 \mathrm{~nm}$. The particle size determined by QELS was around $263 \mathrm{~nm}$ in a monomodal distribution of low polydispersity (Table 1).

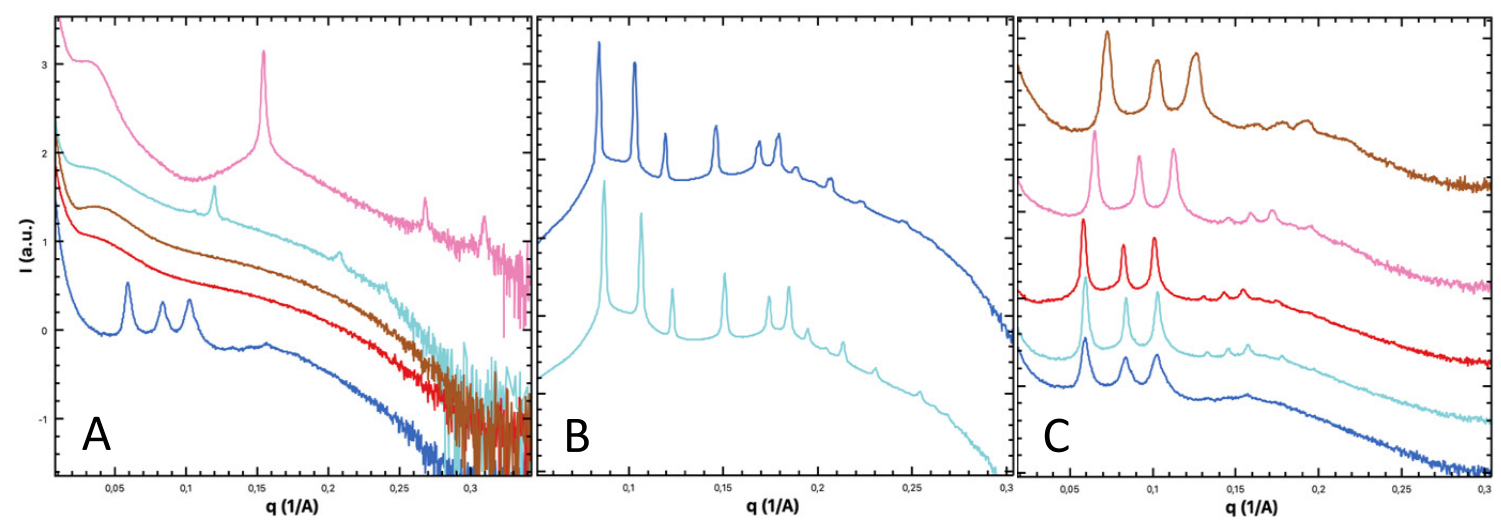

Figure 1. SAXS patterns of MO-based liquid crystalline systems as a function of the Pluronic F127 content and pH. A. Dispersions of self-assembled monoolein/Pluronic F127 systems in pure water (curves from blue to rose: 7\%, 9\%, 11\%, 13\%, 15\% Pluronic F127 (w/w)). B. Bulk phases of pure monoolein in pure water (dark blue) and in a buffer of pH 2.0 (light blue). C. Cubosome dispersions of monoolein/Pluronic F127 (7 wt\%) in pure water (blue) and in buffers of pH 2.0 (light blue), pH 5.0 (red), pH 7.4 (rose) and pH 8.0 (brown). 
The subsequent increment to 9 wt\% and 11 wt\% PF127 considerably changed the liquid crystalline structure of the dispersion as shown by the vanishing Bragg reflections and the appearance of a broad maximum at low scattering vectors. The acquired SAXS patterns evidenced a phase transition occurring with the liquid crystalline organization of the lipid dispersions. The broad maxima may be attributed to intermediate type (cubosome precursors) or sponge-like structures with randomly curved lipid bilayers inside the inner 3D topology. The average particle size increased to 326 and $445 \mathrm{~nm}$, respectively, accompanied by an increment in the size distribution as shown by the standard deviation (Table 1).

Further increase to $13 \mathrm{wt} \%$ and $15 \mathrm{wt} \%$ PF127 content caused a phase transition of the nanoparticles to an inverted hexagonal $\left(\mathrm{H}_{\| 1}\right)$ internal structure. The latter was identified by the Bragg reflections spaced in the ratio 1: V3: V4. Of notice, the 13 wt\% PF127 dispersion presented a lattice parameter $a=5.2 \mathrm{~nm}$, which was reduced to $4.1 \mathrm{~nm}$ at $15 \mathrm{wt} \%$ PF127. In fact, the inverted hexagonal lattice parameter is significantly smaller from that of the cubosome dispersion denoting the smaller internal aqueous channel space for the $H_{\|}$ structure. The size distribution analysis unveiled the presence of two populations of particles in these dispersions (Table 1). The population of larger average size and distribution was attributed to the hexosome nanoparticles, whereas the smaller particles were attributed to vesicles and small cubosomal precursors. These results show that the increment in PF127 leads to transition of the cubosome dispersion to particles of other liquid crystalline organization including hexosomes. Therefore, the $7 \mathrm{wt} \%$ PF127 sample was chosen for further studies with the objective to explore the non-lamellar dispersion as a platform for the development of biopolymer-tailored cubosome particles.

Table 1. Characteristics of the nanoparticle dispersions of monoolein obtained at different concentrations of the Pluronic F127 (PF127) stabilizer.

\begin{tabular}{|c|c|c|c|c|}
\hline $\begin{array}{l}\text { PF127 content } \\
\text { (wt\%) }\end{array}$ & Symmetry & $\begin{array}{c}\text { Lattice } \\
\text { parameter } \\
\begin{array}{c}a \\
(\mathrm{~nm})\end{array}\end{array}$ & $\begin{array}{c}1^{\text {st }} \text { Bragg peak } \\
\text { position } \\
(1 / \AA)\end{array}$ & $\begin{array}{l}\text { Hydrodynamic } \\
\text { diameter } \\
(\mathrm{nm})\end{array}$ \\
\hline 7 & Cubic $/ \mathrm{m} 3 \mathrm{~m}$ & 14.0 & 0.0593 & $263 \pm 28$ \\
\hline 9 & $\begin{array}{c}\text { Cubic phase } \\
\text { precursor } \\
\text { (sponge-like) }\end{array}$ & - & - & $326 \pm 75$ \\
\hline 11 & $\begin{array}{c}\text { Cubic phase } \\
\text { precursor } \\
\text { (sponge-like) }\end{array}$ & - & - & $445 \pm 122$ \\
\hline 13 & $\begin{array}{l}\mathrm{H}_{\|} / \text {Cubic } \\
\text { precursor } \\
\text { coexistence }\end{array}$ & 5.2 & 0.1293 & $\begin{array}{c}634 \pm 145(65 \%) \\
294 \pm 55(35 \%)\end{array}$ \\
\hline 15 & $\begin{array}{l}\mathrm{H}_{\|} / \text {Cubic } \\
\text { precursor } \\
\text { coexistence }\end{array}$ & 4.1 & 0.1551 & $\begin{array}{c}711 \pm 122(66 \%) \\
222 \pm 27(34 \%)\end{array}$ \\
\hline
\end{tabular}




\subsection{Dispersion of bulk lipid cubic phase into cubosome particles in a pH interval}

Figure 1B shows the SAXS profiles for bulk phases of pure monoolein, which was fully hydrated in pure water and in a buffer of $\mathrm{pH} 2.0$ evidencing a prominent $\mathrm{Pn} 3 \mathrm{~m}$ cubic lattice symmetry with the Bragg peaks spaced in the ratios $\sqrt{ } 2: \sqrt{ } 3: \sqrt{ } 4: \sqrt{ } 6: \sqrt{ } 8: \sqrt{ } 9: \sqrt{ } 10: \sqrt{ } 12$. All bulk phases investigated in the $\mathrm{pH}$ range from 2.0 to 8.0 have shown similar profiles (Figure S2). The lattice parameters determined for the bulk phases as a function of $\mathrm{pH}$ are given in Table 2. The data unveil a slight but constant decrease of the inner repeat distances with $\mathrm{pH}$.

The SAXS results obtained for the dispersed MO/PF127 systems are shown in Figure 1C. They confirm the $1 \mathrm{~m} 3 \mathrm{~m}$ cubic symmetry characterizing the inner structure of the cubosomes at all investigated $\mathrm{pH}$ values of the aqueous phase. The lattice parameter increases with lowering the $\mathrm{pH}$ of the aqueous dispersions. Therefore, this evidences the variations of the inner channel diameters within the $I m 3 m$ cubic structure with $\mathrm{pH}$ (Table 2). The observed effect corresponds to shrinking of the hydrated cubosomal structure with a slight reduction of the cubic lattice parameter when $\mathrm{pH}$ increases from acid to alkaline condition.

The QELS measurements showed little variations in the cubosome particle sizes, which were on averages between 247 to $331 \mathrm{~nm}$ (Table 2). At variance, the zeta potential of the dispersions showed $\mathrm{pH}$-dependence under the alkaline conditions. In the acid solution and also in pure water, zeta potential showed close to zero values characteristic of uncharged MO cubosome particles stabilized by PF127. At pH 7.4 and 8.0, the zeta potential reached negative values confirming that the surface of the particles is slightly negatively charged. In the absence of shells formed by associated biopolymer complexes, the negative charge of the MO/PF127 cubosomes might be due to either coordination of phosphate ions from the buffer to the surface of the cubosomes or to partial hydrolysis of MO to oleic acid, which is in an ionized state under alkaline conditions. Moreover, previous studies have discussed the contribution of hydroxide ions adsorption in alkaline solutions [66,67], which can produce a polarized water layer over the surface of the nanoparticles. Such a negative charge contribution was observed for cubosomes produced with nonionic molecules.

Table 2. Characteristics of bulk cubic phases of hydrated monoolein ( $20 \mathrm{wt} \%$ lipid:80 wt\% aqueous phase) in different buffers at varying $\mathrm{pH}$ and of cubosome dispersions stabilized with Pluronic F127 (7 wt\%) in pure water under the same conditions.

\begin{tabular}{|c|c|c|c|c|c|c|}
\hline Phase & $\mathbf{p H}$ & $\begin{array}{c}\text { Cubic } \\
\text { symmetry }\end{array}$ & $\begin{array}{c}\text { Lattice } \\
\text { parameter } \\
\mathbf{a} \\
(\mathbf{n m})\end{array}$ & $\begin{array}{c}\mathbf{1}^{\text {st }} \text { Bragg } \\
\text { peak } \\
\text { position } \\
(\mathbf{1} / \AA)\end{array}$ & $\begin{array}{c}\text { Hydrodynamic } \\
\text { diameter } \\
(\mathbf{n m})\end{array}$ & $\begin{array}{c}\text { Zeta } \\
\text { potential } \\
(\mathbf{m V})\end{array}$ \\
\hline bulk & 2.0 & $P n 3 m$ & 11.2 & 0.0823 & - & - \\
\hline bulk & 5.0 & $P n 3 m$ & 11.1 & 0.0827 & - & - \\
\hline bulk & 7.4 & $P n 3 m$ & 10.8 & 0.0851 & - & - \\
\hline
\end{tabular}




\begin{tabular}{|c|c|c|c|c|c|c|}
\hline bulk & 8.0 & $P n 3 m$ & 10.5 & 0.0876 & - & - \\
\hline bulk & $\begin{array}{c}\text { pure } \\
\text { water }\end{array}$ & $P n 3 m$ & 10.8 & 0.0847 & - & - \\
\hline dispersion & 2.0 & $I m 3 m$ & 13.9 & 0.0599 & $247 \pm 46$ & $0.11 \pm 3.18$ \\
\hline dispersion & 5.0 & $I m 3 m$ & 14.2 & 0.0586 & $298 \pm 56$ & $\begin{array}{c}-0.75 \pm \\
3.74\end{array}$ \\
\hline dispersion & 7.4 & $I m 3 m$ & 12.8 & 0.0653 & $331 \pm 63$ & $\begin{array}{c}-24.7 \pm \\
3.63\end{array}$ \\
\hline dispersion & 8.0 & $I m 3 m$ & 11.4 & 0.0731 & $267 \pm 55$ & $\begin{array}{c}-32.7 \pm \\
4.34\end{array}$ \\
\hline dispersion & $\begin{array}{c}\text { pure } \\
\text { water }\end{array}$ & $\operatorname{Im} 3 m$ & 14.0 & 0.0593 & $263 \pm 28$ & $\begin{array}{c}-1.06 \pm \\
3.45\end{array}$ \\
\hline
\end{tabular}

\subsection{Biopolymer complexes-tailored lipid cubosomes}

3.3.1. Formation of cubosomal nanoassemblies tailored with biopolymer complexes in chitosan- $N$-arginine/alginate shells

Figure 2 shows the SAXS patterns for the variety of cubosome dispersions, produced under different $\mathrm{pH}$ conditions, with self-assembled MO/PF127 (7 wt\%) particles that were subjected to complexes formation. Three concentrations of the biopolymers, namely 10, 15 and $22 \mu \mathrm{M}$, and three proportions of alginate (ALG) relative to chitosan- $N$-arginine (CHarg), 10, 15 and $20 \mathrm{wt} \%$, were included for every preparation. As shown by the SAXS results, all samples present characteristic Bragg reflections of ordered cubic liquid crystalline organization. The Bragg peaks were indexed by the primitive $1 \mathrm{~m} 3 \mathrm{~m}$ cubic symmetry for the majority of samples. The double diamond $\mathrm{Pn} 3 \mathrm{~m}$ inner liquid crystalline organization was present for few ones.

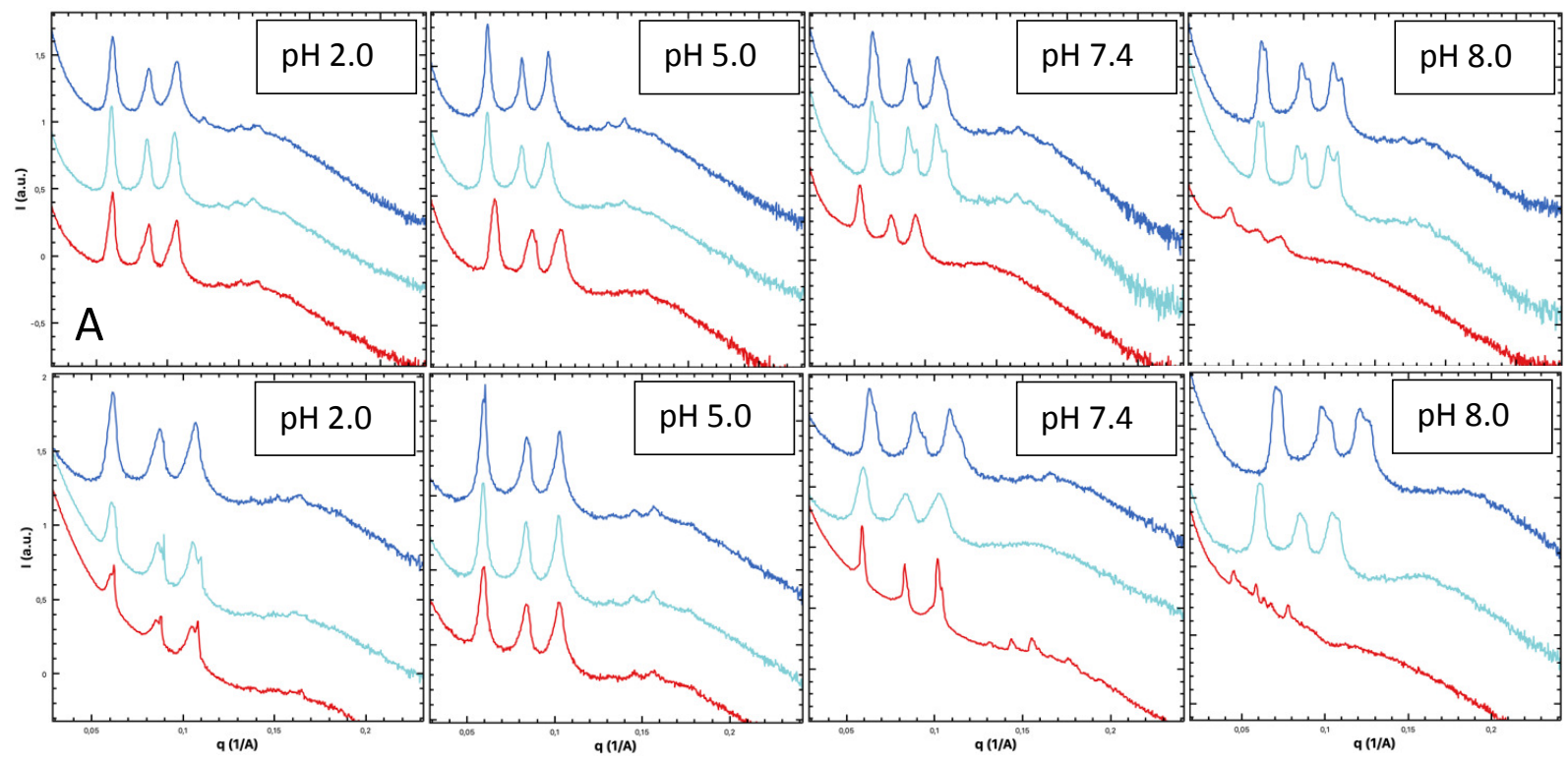


B

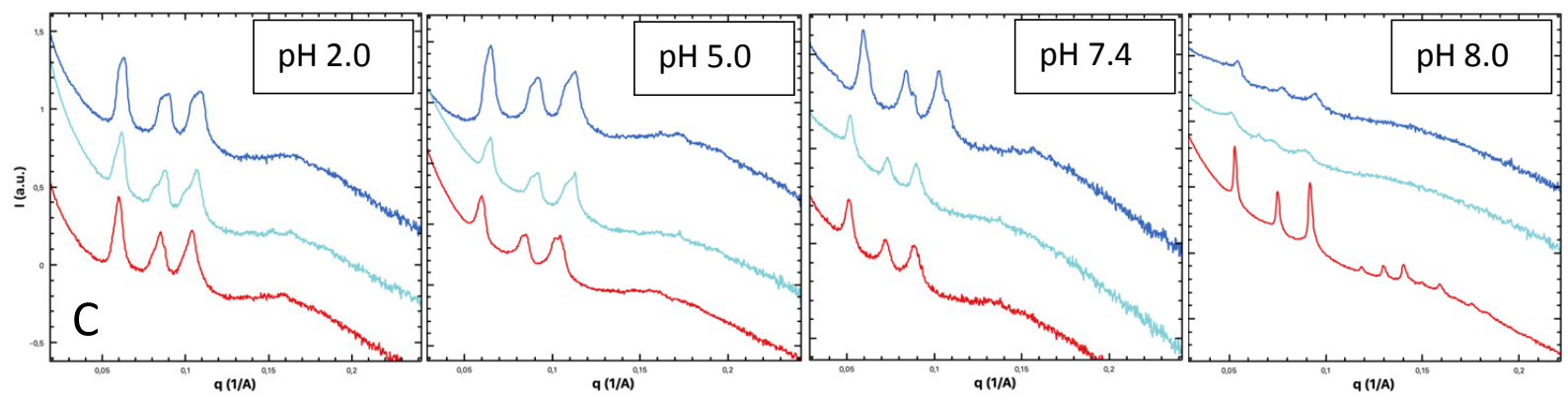

Figure 2. SAXS patterns of biopolymer-tailored monoolein/PF127 (7 wt\%)/chitosan- $N$ arginine (CHarg)/alginate (ALG) cubosome dispersions at different pHs. The plots from up to down are acquired at increasing concentrations of the CHarg-ALG complexes: 10, 15 and 22 $\mu \mathrm{M}$. A. Series of $10 \mathrm{wt} \%$ of ALG relative to CHarg. B. Series of $15 \mathrm{wt} \%$ of ALG relative to CHarg. C. Series of $20 \mathrm{wt} \%$ of ALG relative to $\mathrm{CHarg}$.

As an overall observation, the inclusion of the biopolymers led to changes in the supramolecular organization of the lipid cubosomes. This is evidenced by the appearance of splitted Bragg peaks (Fig. 2A,B) and small but constant modifications of the peak's positions. Moreover, changes in the Bragg peaks relative intensity was noted for some of the samples as well as certain cubic Im3m to Pn3m phase transitions (Fig. 2B,C). These effects can be suggested to be due to changes in the internal aqueous space of the cubic water channel networks with increasing the concentration of the associated biopolymers. The variations of the structural parameters under different $\mathrm{pH}$ conditions are considered in detail bellow. These characteristics all together confirm the feasibility of production of biopolymer-tailored cubosomes, which can be a platform for the development of new hybrid biomaterials for biomedical applications.

\subsection{2. $\mathrm{pH}$-dependence of self-assembled lipid/biopolymer cubosome structures}

Table 3 summarizes the obtained structural results for the biopolymer-tailored cubosomes as a function of $\mathrm{pH}$. A cubic inner organization was present for all investigated compositions of the particles. They were classified in view of the relative proportions between the chitosan- $N$-arginine (CHarg) and alginate (ALG) biopolymers and expressed as \% of ALG.

For every system, the cubic lattice parameter $a$, was calculated from the SAXS results (presented in Figure 2) according to a previously described methodology [11,13]. The values are summarized in Table $\mathbf{3}$ and compared with the data for the cubosome dispersions without biopolymers (Table 2). Under acidic conditions, pH 2.0 and pH 5.0, the cubic lattice parameters are between 13 and $14 \mathrm{~nm}$. However, under alkaline conditions $(\mathrm{pH} 7.4$ and $\mathrm{pH}$ 8.0), more significant peculiarities were unveiled. First, a decrease of the lattice parameter observed for the pure MO/PF127 cubosomes in alkaline solutions was not found. Instead, splitted Bragg peaks appeared for the majority of the biopolymer-tailored cubosomes at 
increasing $\mathrm{pH}$. The lattice parameter was not $\mathrm{pH}$-dependent at low $\mathrm{pH}$. Essentially, the lattice parameter increased for the samples containing higher concentration of biopolymers and at the higher proportion of ALG (20\% ALG). Under acidic conditions, splitted peaks were observed at pH 2.0 only for $15 \% \mathrm{ALG}$ at $15 \mu \mathrm{M}$ and $22 \mu \mathrm{M}$ biopolymer concentrations.

As a general trend, the lattice parameters increased more significantly for the $22 \mu \mathrm{M}$ biopolymer concentration. Notably, a phase transition from the cubic Im3m to cubic Pn3m topology was established with these samples at $\mathrm{pH} 8.0$ for the $10 \%$ and $15 \%$ ALG content as well as for the $15 \mu \mathrm{M}$ biopolymers concentration with 20\% ALG. The determined lattice parameter for the $\mathrm{Pn} 3 \mathrm{~m}$ phase structure increased at pH 8.0, contrary to those of the bulk phases and the dispersions free of biopolymers (Table $\mathbf{2}$ ).

These structural effects present evidence of the partial swelling of the cubic unit cells, which suggests that the biopolymers are likely to be partially inserted in the internal structure of the cubosomes. The hydrophobic chains of chitosan may penetrate the lipid/water interfaces, whereas the charged residues may partition into the aqueous channel networks. The samples containing $22 \mu \mathrm{M}$ biopolymers with $20 \%$ ALG deserve further consideration. While a phase transition to Pn3m structure was not observed, a sharpening of the Bragg peaks and increased relative peak intensity was established at $\mathrm{pH} 8.0$ (Figure 2). The calculated lattice parameter for this sample was $15.67 \mathrm{~nm}$, thus smaller compared to the value of $17.31 \mathrm{~nm}$ determined at $\mathrm{pH} 7.4$ (Table 3). The sharpening of the Bragg reflections evidences a longer-range order of the liquid crystalline structure. Along with the cubic unit cell reduction, the results suggest that the biopolymers are partially inserted in the cubic lattice networks of the particles. If expelled from the lipid/water interfaces and the internal channel networks, the biopolymer chains might remain associated over the external surface of the cubosomes. In any case, the SAXS data reveal that the biopolymers provide stable and well-defined liquid crystalline structures under the investigated conditions.

The QELS size profiles, expressed as hydrodynamic diameters, revealed further features of the biopolymer-tailored cubosome dispersions. As shown in Table 3, the majority of the samples presented a bimodal nanoparticle size distribution for the $10 \%$ ALG series at $\mathrm{pH} 2.0$, 7.4 and 8.0 , but monomodal size distribution at $\mathrm{pH}$ 5.0. For the $15 \%$ and $20 \%$ ALG series, the bimodal distribution was observed under the alkaline conditions $(\mathrm{pH} 7.4$ and 8.0), but not in the acidic solutions (pH 2.0 and 5.0). Upon analyzing the data in detail, the bimodal distribution was found to be comprised by a higher proportion ( 70\%, QELS intensity weighted) of larger particles, e.g. 500-900 nm with higher polydispersity. A relative smaller proportion ( $30 \%)$ of size-reduced particles ranging around 140-230 nm and with lower polydispersity was found for the samples at $\mathrm{pH}$ 7.4. However, a turnover in the particles size profile was found at $\mathrm{pH} 8.0$ with random alterations in the proportions between the two particles populations, but most importantly, a reduction in the average sizes for the largest proportion. 


\subsubsection{Zeta potential variations of cubosomal lipid particles tailored with biopolymer complexes}

The zeta potential data equally shown in Table $\mathbf{3}$ provide further information, not only about the particles surface charge, but complementary structure information if associated to the QELS size profiles. As a general characteristic, zeta potential was nearly neutral to moderately positive for the particle dispersions in the acidic solutions, but increasingly negative in the alkaline solutions. For the $10 \%$ ALG series, zeta potential increased with the concentration of the biopolymers at $\mathrm{pH} 2.0$, but only for the higher concentration at $\mathrm{pH} 5.0$. This increase was less expressive for the $15 \%$ ALG series and even less for the $20 \%$ ALG series. Thereby, the relative concentration of ALG plays a crucial role for the surface characteristics of the particles.

Additionally, partial aggregation of the cubosomes was observed at $\mathrm{pH} 2.0$ for the $10 \% \mathrm{ALG}$ series with $10 \mu \mathrm{M}$ and $15 \mu \mathrm{M}$ of biopolymer complexes, as established by the size measurements (a significant population of particles with sizes around 500 and $800 \mathrm{~nm}$ was present). For these samples the amount and the proportion between the biopolymers was not enough to avoid the partial aggregation, which did not occur for all other samples at $\mathrm{pH}$ 2.0 and 5.0. Although the zeta potential was slightly positive, i.e. 4.95 and $8.35 \mathrm{mV}$ on average, it was not enough to provide charge repulsion between the particles, leading to their partial aggregation. Despite the two populations of particles, the zeta potential showed a single charge profile for all samples. This indicated that both larger and smaller nanoscale structures (which are due to the sonication-vortex fabrication process) carry similar surface charge characteristics and thereby the larger particles actually result from partial aggregation of the smaller ones. Alternatively, the small particles appear to be nonequilibrium fragments of vesicular membranes from the bigger nanoparticles [68].

It is interesting to note that the zeta potential values at $\mathrm{pH} 5.0$ were not far from the neutrality for the majority of samples with 10 and $15 \mu \mathrm{M}$ at 10 and $15 \%$ ALG and as well for the $22 \mu \mathrm{M}$ at 20\% ALG. Despite, it increased to 22.9 and $16.2 \mathrm{mV}$ on average for the $22 \mu \mathrm{M}$ at 10 and $15 \%$ ALG. These results evidence the zeta potential dependency on the biopolymers concentration and ALG proportion. The absence of aggregation and the production of biopolymer-tailored cubosomes with monomodal distribution and similar size for all the samples at $\mathrm{pH} 5.0$ should be attributed to the charge balance between the opposite charged biopolymers as discussed below.

The zeta potential results were all negative for the samples at $\mathrm{pH} 7.4$ and 8.0. In terms of biopolymers concentration dependence, the zeta potential decreased slightly with the concentration increase and the highest negative values were obtained for all $22 \mu \mathrm{M}$ samples. However, the variation in ALG\% had no marked effect on the zeta potential in both alkaline solutions as noticeable if comparing the results of the corresponding samples in Table 3.

It is apparent that the surface charge of the particles in these solutions was not negative enough to avoid the partial aggregation of the biopolymer-tailored cubosomes. The majority of samples showed the presence of two populations of nanoparticles, for which the single zeta potential profile denoted similar surface charge characteristics for both particle 
topologies. The partial aggregation was less pronounced considering the proportions between the two particle populations, in $\mathrm{pH} 8.0$ compared to $\mathrm{pH} 7.4$, which can be related to the higher negative zeta potentials found at $\mathrm{pH}$ 8.0. Hence, the increase in surface negative charge on the particles must have contributed in diminishing the aggregation. This is consistent if considering the $22 \mu \mathrm{M}$ samples at 10 and $15 \%$ ALG in pH 8.0, which showed higher negative zeta potential of -37.4 and $-35.7 \mathrm{mV}$ on average and for which no aggregation occurred. Instead, the particles presented similar size profile as the respective ones in $\mathrm{pH}$ 5.0, which showed positive zeta potential, 22.9 and $16.2 \mathrm{mV}$ on average, respectively.

Table 3

Table 3. Structural characteristics (cubic lattice parameters), hydrodynamic diameters and zeta potentials at different $\mathrm{pH}$ of lipid/biopolymer cubosome dispersions monoolein/PF127 (7 wt\%)/chitosan- $N$-arginine ( $\mathrm{CHarg}$ )/ALG for three concentrations of the polysaccharides and 10,15 and 20 wt\% alginate (ALG) content relative to $\mathrm{CHarg}$.

\subsubsection{Charge transitions in the biopolymer complexes imparting $\mathrm{pH}$-responsiveness of the cubosome particles}

The $\mathrm{pH}$-responsiveness and the structural features of the biopolymer-tailored cubosomes were considered by analyzing the surface charge characteristics, which differ under every $\mathrm{pH}$ condition. Figure 3 shows the zeta potential variation as a function of $\mathrm{pH}$ for the dispersions with $22 \mu \mathrm{M}$ of biopolymers at different proportions of ALG and compared to the cubosome dispersion without biopolymers. The higher concentration of $22 \mu \mathrm{M}$ was chosen to better evidence the biopolymers effects, although the 10 and $15 \mu \mathrm{M}$ samples presented a similar tendency. The plot evidences a progressive increase of zeta potential to positive values under acidic conditions and to negative values under alkaline conditions with reduction of ALG proportion relative to CHarg. Hence, it is apparent that the $10 \%$ ALG proportion (red spheres) provides the higher $\mathrm{pH}$-responsiveness to the studied particles, since zeta potential relays around $20 \mathrm{mV}$ in the acid solutions and drops to nearly $-40 \mathrm{mV}$ in the alkaline region. 


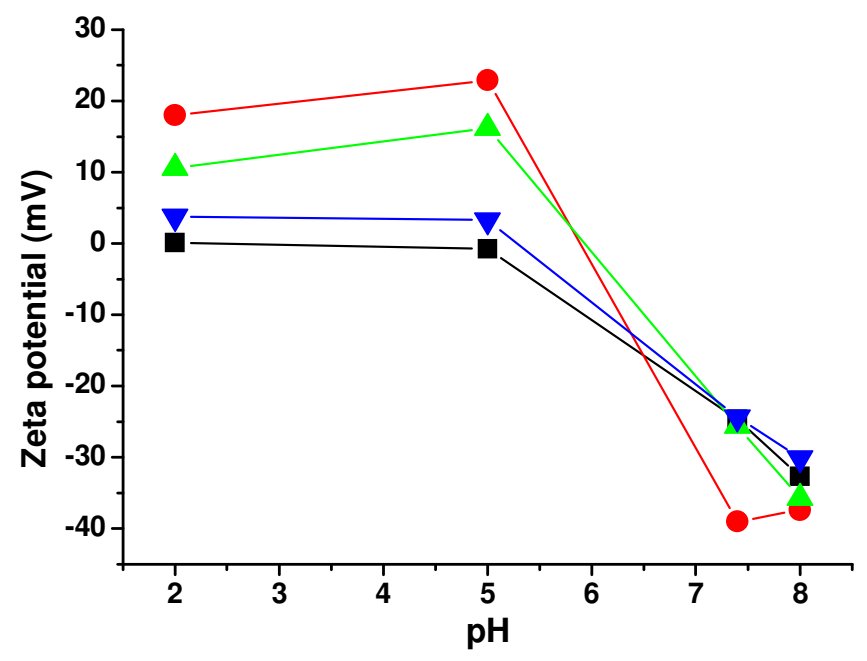

Figure 3. Zeta potential variations as a function of $\mathrm{pH}$ for cubosomes prepared with $22 \mu \mathrm{M}$ CHarg-ALG biopolymer complexes at proportions of $10 \mathrm{wt} \%$ (red sphere), $15 \mathrm{wt} \%$ (green triangle) and $20 \mathrm{wt} \%$ (blue triangle) of ALG relative to CHarg. The data are compared with the zeta potentials of cubosomes without biopolymer shells (black squares). The lines are guide to the eye.

These results can be related to the ionization state of the biopolymers at every $\mathrm{pH}$, which establishes the charge equilibrium between the polyelectrolytes CHarg and ALG [69]. In pH 2.0, CHarg is completely protonated on amine and guanidinium groups, thus providing a polycation structure. However, ALG (pKa 3.38 (mannuronate residues) and 3.65 (guluronate residues)) is in the alginic acid form, i.e., with hydrogen in the carboxylic acid groups, rendering the structure in the neutral state. This scenario renders the cubosomes surface positively charged when the proportion of ALG is low. It seems that the surface charge tends to neutralization, when increasing this proportion to $20 \%$, not because of the charge equilibrium but rather due to conformation characteristics of the ALG chains (insoluble in strong $\mathrm{pH}$ and whose outcome effectively diminishes the polycation charge contribution of CHarg on the cubosomes surface). This effect has been previously suggested for plain chitosan and ALG nanoparticles at 50:50 w:w proportion [56] and for CHarg and ALG nanoparticles at $9 \mathrm{wt} \%$ ALG [54].

In pH 5.0, the carboxylic acid groups of ALG are ionized leading to formation of a polyanion structure [69]. Thus, the charge equilibrium between CHarg and ALG is partially established and indeed the equilibrium increases with the increase of ALG proportion, which is demonstrated by the respective reduction of zeta potential for the $20 \mathrm{wt} \% \mathrm{ALG}$. Thereby, the positive surface charge of the biopolymer-tailored cubosomes increases under acid conditions when the ALG proportion decreases.

In pH 7.4 and 8.0, the CHarg suffers deprotonation of the amine groups (pKa 6), but not on the few guanidinium groups of bonded arginine (pKa 13, [70]). Thus, the CHarg chains present an average of $3.5 \%$ of cationic monomers (considering the amount of linked 
arginine). The ALG chains remain completely negatively charged. Since the low proportion of the positively charged monomers of $\mathrm{CHarg}$ is always completely neutralized by all the used proportions of ALG, the resulting zeta potential relays on similar negative values for the majority of samples under these conditions. A remark is given to the $10 \%$ ALG with a stronger negative zeta potential in $\mathrm{pH} 7.4$, which suggests that the conformation between the biopolymers favored the predominance of the free carboxylic groups of ALG on the surface of the cubosomes and over the CHarg.

The described $\mathrm{pH}$-responsiveness, related to the structural features of the biopolymertailored cubosomes, may represent important advantages for the development of colloidal vehicles for biomedical applications where $\mathrm{pH}$ variation plays an important role. Previously, the combination of $\mathrm{CHarg}$ and ALG in the production of micro and nanoparticles as drug delivery systems has shown great potential for in vivo oral administration [54,56-59]. Indeed, the gastrointestinal tract presents extreme $\mathrm{pH}$ variation to which drug delivery devices can be developed to respond in a manner to provide localized, optimized, and prolonged delivery of the carried bioactives. Regarding the cubosomes, the internal crystalline structure provides a complex crystalline network for the entrapment and encapsulation of hydrophobic and hydrophilic molecules [71]. With the intrinsic slow disassembling of the crystalline structure in biological media [14], the cubosomes may provide a prolonged release of the transported bioactives. However, plain cubosomes may be removed from the gastrointestinal tract before accomplishing the drug delivery purpose. Therefore, the development of biopolymer-tailored cubosomes offers a new direction in the application of cubosomes in oral drug delivery [17].

The surface charge profiles, provided by CHarg and ALG in the generated complexes in this study, show that the biopolymer-tailored cubosomes have reduced size and slightly positive surface charge in strong acid media, which is typically found in the stomach (Figure 4). In the intestinal tract, the $\mathrm{pH}$ shows a gradual increase from around 6.0 in the duodenum to 7.4 in the terminal ileum. In a previous study, performed with nanoparticles produced with the same CHarg and ALG [54], the zeta potential at $\mathrm{pH} 6.5$ varied between -8 and $-20 \mathrm{mV}$. These previous results for the biopolymers zeta potential, if placed in Figure 3, would be found around the guide lines between $\mathrm{pH} 5.0$ and 7.4, reinforcing the observation that the biopolymer-tailored cubosomes show a decreasing zeta potential tendency with $\mathrm{pH}$ increase, as shown in this study. When passing to the slightly alkaline media, typical of intestine condition, the surface charge reverses to negative and the particles tend to aggregate. This aggregation process can be profitable to provide prolonged permanence of the structures in the intestinal tract. It may improve their retention at the mucosa, hence avoiding the rapid metabolic removal with mucoadhesion, which can be achieved by interactions of the polysaccharides with proteins of the intestinal mucosal layers $[54,72,73]$. The improved retention in intestines can optimize a prolonged delivery in the organ where absorption of bioactives is directed. Thereby, the assembly of lipid cubosomes with polyelectrolyte complexes, providing $\mathrm{pH}$-responsive structural characteristics, represents a means to safely transport encapsulated bioactives through the strong acid in stomach besides preventing 
enzymatic degradation in the small intestine. Furthermore, the mucoadhesive property of the biopolymers can render stronger interaction and deeper penetration of the nanodevices in the mucosal layers of intestine, hence ensuring prolonged retention, and thus rendering prolonged release of the bioactives directly to the absorptive columnar epithelium of intestine, promoting the absorption and improving the bioavailability [74]. Therefore, the CHarg and ALG macromolecules on the surface of cubosomes provide a means to improve the mucoadhesion, thus rendering the biopolymer-tailored cubosomes with profitable properties for the development as vehicles for oral delivery of bioactives.

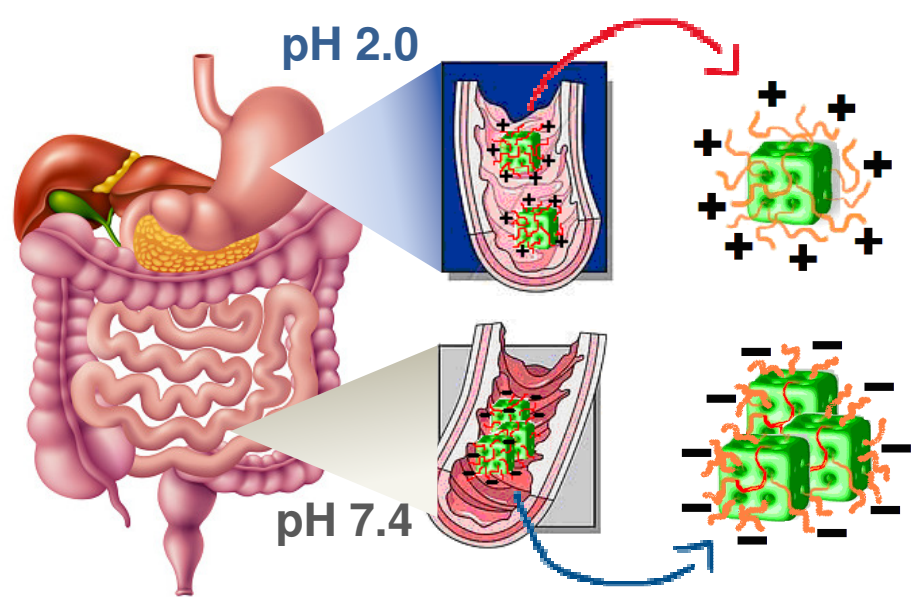

Figure 4. Representation of the human gastrointestinal tract with an indication of the $\mathrm{pH}$ zones in the stomach and the intestine, for which the biopolymer-tailored cubosome nanoparticles assume a positive net surface charge $(\mathrm{pH} 2.0)$ and aggregated assemblies with a negative net surface charge. These characteristics may improve the mucoadhesion to the intestinal mucosal layers.

\section{Conclusion}

Biopolymer-tailored cubosomes with $\mathrm{pH}$ sensitivity offer a new direction in the application of cubosome carriers in oral drug delivery. Here hybrid cubosome particles tailored with two biopolymers were produced with an optimized concentration of the amphiphilic stabilizer Pluronic F127 and with various $\mathrm{N}$-arginine-modified chitosan and alginate relative proportions in the formed polyelectrolyte complexes at different $\mathrm{pH}$ values. The liquid crystalline structure and relative stability of the colloidal dispersions was evidenced in a range of $\mathrm{pH}$ conditions, characteristic of the human gastrointestinal tract. The structural changes established by SAXS suggested that the biopolymers were successfully incorporated to the cubosomal lipid structures, rendering the obtained nanoparticles $\mathrm{pH}$-responsive vehicles. Both biopolymers have been incorporated in different types of nanoparticles $[44,54,57]$. However, they built-up a promising new nanostructured material in association with lipid-based cubosomes by tailoring liquid crystalline structure and poly-charged shells, which can render stronger interaction and deeper penetration in the mucosal layers of 
intestine. Hence, these characteristics of the lipid-biopolymer cubosomes may be explored for the development of oral drug delivery systems for bioactive compounds. In this context, further directions could drive to the investigation of the entrapment and encapsulation potential of the hybrid cubosomes for hydrophilic and hydrophobic molecules, besides in vitro release and in vivo intestinal permeability studies and resulting bioavailability profile of the delivered bioactives.

\section{CRediT authorship contribution statement}

Patrick D. Mathews: Methodology, Investigation, Data curation, Formal analysis. Omar Mertins: Conceptualization, Methodology, Investigation, Data curation, Formal analysis, Supervision, Writing - original draft preparation, Writing - review \& editing, Funding acquisition. Borislav Angelov: Methodology, Data curation, Formal analysis, Validation, Funding acquisition. Angelina Angelova: Conceptualization, Methodology, Investigation, Data curation, Formal analysis, Writing - original draft preparation, Writing - review \& editing, Supervision.

\section{Declaration of Competing Interest}

The authors declare that they have no known competing financial interests or personal relationships that could have appeared to influence the work reported in this article.

\section{Acknowledgements}

The authors gratefully acknowledge the allocation of beam time at Synchrotron SOLEIL (Saint Aubin, France) through the project 20191836 and the scientific and technical support of Dr. T. Bizien at the SWING beamline. P.D.M. thanks the Sao Paulo Research Foundation (FAPESP) for the postdoctoral internship (19/13147-6). O.M. thanks FAPESP for the internship project (18/21727-0) and for the research financial support (21/00971-2). B.A. obtained financial support from the collaborative project with JINR, Dubna, Russia (3+3 program, No. 204, item 27 from 25.03.2020) and the projects "Structural Dynamics of Biomolecular Systems" (ELIBIO) (CZ.02.1.01/0.0/0.0/15_003/0000447) and "Advanced research using high-intensity laser produced photons and particles" (CZ.02.1.01/0.0/0.0/16_019/0000789) from the European Regional Development Fund. A.A. acknowledges a membership in the CNRS GDR2088 BIOMIM research network.

\section{Appendix A. Supplementary data}

Supplementary data to this article can be found online at

\section{References}

1. Hussain N, Jaitley V, Florence AT. Recent advances in the understanding of uptake of microparticulates across the gastrointestinal lymphatics. Adv Drug Deliv Rev. 2001;50(1-2):107-142. doi:10.1016/s0169-409x(01)00152-1 
2. Fong W-K, Negrini R, Vallooran JJ, Mezzenga R, Boyd BJ. Responsive self-assembled nanostructured lipid systems for drug delivery and diagnostics. J Colloid Interface Sci. 2016;484:320-339. doi:https://doi.org/10.1016/j.jcis.2016.08.077

3. Angelova A, Garamus VM, Angelov B, Tian Z, Li Y, Zou A. Advances in structural design of lipid-based nanoparticle carriers for delivery of macromolecular drugs, phytochemicals and anti-tumor agents. Adv Colloid Interface Sci. 2017;249:331-345. doi:https://doi.org/10.1016/j.cis.2017.04.006

4. Kim TH, Kwon K, Yoo DS, et al. Monoolein cubic phase containing alginate/cystamine gel for controlled release of epidermal growth factor. J Dispers Sci Technol. 2019;40(1):119-127. doi:10.1080/01932691.2018.1467325

5. Murgia S, Biffi S, Mezzenga R. Recent advances of non-lamellar lyotropic liquid crystalline nanoparticles in nanomedicine. Curr Opin Colloid Interface Sci. 2020;48:2839. doi:https://doi.org/10.1016/j.cocis.2020.03.006

6. Rahanyan-Kägi N, Aleandri S, Speziale C, Mezzenga R, Landau EM. Stimuli-Responsive Lipidic Cubic Phase: Triggered Release and Sequestration of Guest Molecules. Chem A Eur J. 2015;21(5):1873-1877. doi:https://doi.org/10.1002/chem.201405580

7. Park SH, Kim J-C. Complexation-responsive monoolein cubic phase containing extract of Bambusae Caulis in Taeniam. Int J Polym Mater Polym Biomater. 2020;69(1):44-52. doi:10.1080/00914037.2018.1539986

8. Tan A, Hong L, Du JD, Boyd BJ. Self-Assembled Nanostructured Lipid Systems: Is There a Link between Structure and Cytotoxicity? Adv Sci. 2019;6(3). doi:10.1002/advs.201801223

9. Yaghmur A, Al-Hosayni S, Amenitsch H, Salentinig S. Structural Investigation of Bulk and Dispersed Inverse Lyotropic Hexagonal Liquid Crystalline Phases of Eicosapentaenoic Acid Monoglyceride. Langmuir. 2017;33(49):14045-14057. doi:10.1021/acs.langmuir.7b03078

10. Li Y, Angelova A, Hu F, et al. pH Responsiveness of Hexosomes and Cubosomes for Combined Delivery of Brucea javanica Oil and Doxorubicin. Langmuir. 2019;35(45):14532-14542. doi:10.1021/acs.langmuir.9b02257

11. Rakotoarisoa M, Angelov B, Espinoza S, Khakurel K, Bizien T, Angelova A. Cubic Liquid Crystalline Nanostructures Involving Catalase and Curcumin: BioSAXS Study and Catalase Peroxidatic Function after Cubosomal Nanoparticle Treatment of Differentiated SH-SY5Y Cells. Mol. 2019;24(17). doi:10.3390/molecules24173058

12. Karami Z, Hamidi M. Cubosomes: remarkable drug delivery potential. Drug Discov Today. 2016;21(5):789-801. doi:https://doi.org/10.1016/j.drudis.2016.01.004

13. Angelov B, Angelova A, Drechsler M, Garamus VM, Mutafchieva R, Lesieur S. Identification of large channels in cationic PEGylated cubosome nanoparticles by synchrotron radiation SAXS and Cryo-TEM imaging. Soft Matter. 2015;11(18):36863692. doi:10.1039/C5SM00169B

14. Angelova A, Angelov B, Drechsler M, Lesieur S. Neurotrophin delivery using nanotechnology. Drug Discov Today. 2013;18(23):1263-1271. doi:https://doi.org/10.1016/j.drudis.2013.07.010

15. Salentinig S, Tangso KJ, Hawley A, Boyd BJ. pH-Driven Colloidal Transformations Based on the Vasoactive Drug Nicergoline. Langmuir. 2014;30(49):14776-14781. doi:10.1021/la503824z

16. Sethuraman V, Janakiraman K, Krishnaswami V, Natesan S, Kandasamy R. pH responsive delivery of lumefantrine with calcium phosphate nanoparticles loaded 
lipidic cubosomes for the site specific treatment of lung cancer. Chem Phys Lipids. 2019;224:104763. doi:10.1016/j.chemphyslip.2019.03.016

17. Mertins O, Mathews PD, Angelova A. Advances in the Design of pH-Sensitive Cubosome Liquid Crystalline Nanocarriers for Drug Delivery Applications. Nanomater (Basel, Switzerland). 2020;10(5):963. doi:10.3390/nano10050963

18. Tilley AJ, Drummond CJ, Boyd BJ. Disposition and association of the steric stabilizer Pluronic ${ }^{\circledR}$ F127 in lyotropic liquid crystalline nanostructured particle dispersions. J Colloid Interface Sci. 2013;392:288-296. doi:10.1016/j.jcis.2012.09.051

19. Chong JYT, Mulet X, Keddie DJ, et al. Novel Steric Stabilizers for Lyotropic Liquid Crystalline Nanoparticles: PEGylated-Phytanyl Copolymers. Langmuir. 2015;31(9):2615-2629. doi:10.1021/la501471z

20. Angelova A, Angelov B, Drechsler M, Garamus VM, Lesieur S. Protein entrapment in PEGylated lipid nanoparticles. Int J Pharm. 2013;454(2):625-632. doi:10.1016/j.ijpharm.2013.06.006

21. Nilsson C, Østergaard J, Larsen SW, Larsen C, Urtti A, Yaghmur A. PEGylation of phytantriol-based lyotropic liquid crystalline particles--the effect of lipid composition, PEG chain length, and temperature on the internal nanostructure. Langmuir. 2014;30(22):6398-6407. doi:10.1021/la501411w

22. Liu Z, Yu L, Gu P, et al. Surface-Engineered Cubosomes Serve as a Novel Vaccine Adjuvant to Modulate Innate Immunity and Improve Adaptive Immunity in vivo. Int J Nanomedicine . 2020;15:8595-8608. doi:10.2147/IJN.S266165

23. Park SH, Kim J-C. Oxidation-responsive cubic phase incorporating poly(hydroxyethyl acrylamide-co-phenyl vinyl sulfide). Colloid Polym Sci. 2019;297(1):23-34. doi:10.1007/s00396-018-4437-1

24. Park D, Park SH, Kim J-C. In vitro Anti-cancer Efficacy and Cellular Interaction of Cubic Phases Containing Cinnamic Acid, Poly(ethyleneimine), and Doxorubicin. Biotechnol Bioprocess Eng. 2020;25(2):235-245. doi:10.1007/s12257-019-0123-5

25. Kumar M, Patil N, Ambade A V, Kumaraswamy G. Large PAMAM Dendron Induces Formation of Unusual P4332 Mesophase in Monoolein/Water Systems. Langmuir. 2018;34(23):6827-6834. doi:10.1021/acs.langmuir.8b00551

26. Deshpande S, Venugopal E, Ramagiri S, Bellare JR, Kumaraswamy G, Singh N. Enhancing Cubosome Functionality by Coating with a Single Layer of Poly- $\varepsilon$-lysine. ACS Appl Mater Interfaces. 2014;6(19):17126-17133. doi:10.1021/am5047872

27. Driever CD, Mulet X, Waddington LJ, et al. Layer-by-Layer Polymer Coating on Discrete Particles of Cubic Lyotropic Liquid Crystalline Dispersions (Cubosomes). Langmuir. 2013;29(42):12891-12900. doi:10.1021/la401660h

28. Driever CD, Mulet X, Johnston APR, et al. Converging layer-by-layer polyelectrolyte microcapsule and cubic lyotropic liquid crystalline nanoparticle approaches for molecular encapsulation. Soft Matter. 2011;7(9):4257-4266. doi:10.1039/COSM01410A

29. Svensson O, Thuresson K, Arnebrant T. Interactions between chitosan-modified particles and mucin-coated surfaces. J Colloid Interface Sci. 2008;325(2):346-350. doi:10.1016/j.jcis.2008.06.013

30. Verma P, Ahuja M. Optimization, characterization and evaluation of chitosan-tailored cubic nanoparticles of clotrimazole. Int J Biol Macromol. 2015;73:138-145. doi:https://doi.org/10.1016/j.ijbiomac.2014.10.065

31. Wei $Y$, Zhang J, Zheng $Y$, et al. Cubosomes with surface cross-linked chitosan exhibit 
sustained release and bioavailability enhancement for vinpocetine. RSC Adv. 2019;9(11):6287-6298. doi:10.1039/C8RA10302J

32. Ruocco N, Frielinghaus $\mathrm{H}$, Vitiello $\mathrm{G}$, et al. How hydrophobically modified chitosans are stabilized by biocompatible lipid aggregates. J Colloid Interface Sci. 2015;452:160-168. doi:10.1016/j.jcis.2015.03.058

33. Kwon TK, Kim J-C. pH-Dependent Release from Monoolein Cubic Phase Containing Hydrophobically Modified Chitosan. J Dispers Sci Technol. 2011;32(4):480-484. doi:10.1080/01932691003756761

34. Sharma S, Bisht A, Tewari S, Dave V. Recent Advances in Polymer-Modified Liposomes for Cancer Treatment. Nanopharmaceutical Adv Deliv Syst. Published online January 12, 2021:379-405. doi:https://doi.org/10.1002/9781119711698.ch17

35. Moosavian SA, Sahebkar A. Aptamer-functionalized liposomes for targeted cancer therapy. Cancer Lett. 2019;448:144-154. doi:10.1016/j.canlet.2019.01.045

36. Miyazaki M, Yuba E, Hayashi H, Harada A, Kono K. Hyaluronic Acid-Based pH-Sensitive Polymer-Modified Liposomes for Cell-Specific Intracellular Drug Delivery Systems. Bioconjug Chem. 2018;29(1):44-55. doi:10.1021/acs. bioconjchem.7b00551

37. Yamazoe E, Fang J-Y, Tahara K. Oral mucus-penetrating PEGylated liposomes to improve drug absorption: Differences in the interaction mechanisms of a mucoadhesive liposome. Int J Pharm. 2021;593:120148. doi:10.1016/j.ijpharm.2020.120148

38. Shtenberg $\mathrm{Y}$, Goldfeder $\mathrm{M}$, Prinz $\mathrm{H}$, et al. Mucoadhesive alginate pastes with embedded liposomes for local oral drug delivery. Int J Biol Macromol. 2018;111:62-69. doi:10.1016/j.ijbiomac.2017.12.137

39. Klemetsrud T, Kjøniksen A-L, Hiorth M, Jacobsen J, Smistad G. Polymer coated liposomes for use in the oral cavity - a study of the in vitro toxicity, effect on cell permeability and interaction with mucin. J Liposome Res. 2018;28(1):62-73. doi:10.1080/08982104.2016.1255640

40. Gómez-Guillén MC, Montero MP. Enhancement of oral bioavailability of natural compounds and probiotics by mucoadhesive tailored biopolymer-based nanoparticles: A review. Food Hydrocoll. 2021;118:106772. doi:https://doi.org/10.1016/j.foodhyd.2021.106772

41. Ling Tan JS, Roberts CJ, Billa N. Mucoadhesive chitosan-coated nanostructured lipid carriers for oral delivery of amphotericin B. Pharm Dev Technol. 2019;24(4):504-512. doi:10.1080/10837450.2018.1515225

42. Rahat I, Rizwanullah M, Gilani SJ, et al. Thymoquinone loaded chitosan - Solid lipid nanoparticles: Formulation optimization to oral bioavailability study. J Drug Deliv Sci Technol. 2021;64:102565. doi:https://doi.org/10.1016/j.jddst.2021.102565

43. Saesoo S, Bunthot S, Sajomsang W, et al. Phospholipid-chitosan hybrid nanoliposomes promoting cell entry for drug delivery against cervical cancer. J Colloid Interface Sci. 2016;480:240-248. doi:10.1016/j.jcis.2016.06.071

44. Garcia BBM, Mertins O, Silva ER da, Mathews PD, Han SW. Arginine-modified chitosan complexed with liposome systems for plasmid DNA delivery. Colloids Surfaces $B$ Biointerfaces. 2020;193:111131. doi:https://doi.org/10.1016/j.colsurfb.2020.111131

45. Mertins O, Lobo SE, Mathews PD, Han SW. Interaction of pDNA with reverse phase chitosome. Colloids Surfaces A Physicochem Eng Asp. 2018;543:76-82. doi:https://doi.org/10.1016/j.colsurfa.2018.02.005

46. Marón LB, Covas CP, da Silveira NP, et al. LUVs Recovered with Chitosan: A New 
Preparation for Vaccine Delivery. J Liposome Res. 2007;17(3-4):155-163. doi:10.1080/01460860701525444

47. Alavi S, Haeri A, Dadashzadeh S. Utilization of chitosan-caged liposomes to push the boundaries of therapeutic delivery. Carbohydr Polym. 2017;157:991-1012. doi:https://doi.org/10.1016/j.carbpol.2016.10.063

48. Caddeo C, Díez-Sales O, Pons R, et al. Cross-linked chitosan/liposome hybrid system for the intestinal delivery of quercetin. J Colloid Interface Sci. 2016;461:69-78. doi:10.1016/j.jcis.2015.09.013

49. Wang M, Liu M, Xie T, Zhang B-F, Gao X-L. Chitosan-modified cholesterol-free liposomes for improving the oral bioavailability of progesterone. Colloids Surf $B$ Biointerfaces. 2017;159:580-585. doi:10.1016/j.colsurfb.2017.08.028

50. Mertins O, Dimova R. Insights on the interactions of chitosan with phospholipid vesicles. Part I: Effect of polymer deprotonation. Langmuir. 2013;29(47):14545-14551. doi:10.1021/la403218c

51. Mertins O, Lionzo MIZ, Micheletto YMS, Pohlmann AR, Silveira NP da. Chitosan effect on the mesophase behavior of phosphatidylcholine supramolecular systems. Mater Sci Eng C. 2009;29(2):463-469. doi:https://doi.org/10.1016/j.msec.2008.08.038

52. Mertins $O$, Mathews PD, Gomide AB, Baptista MS, Itri R. Effective protection of biological membranes against photo-oxidative damage: Polymeric antioxidant forming a protecting shield over the membrane. Biochim Biophys Acta. 2015;1848(10 Pt A):2180-2187. doi:10.1016/j.bbamem.2015.06.005

53. Mertins O, da Silveira NP, Pohlmann AR, Schröder AP, Marques CM. Electroformation of giant vesicles from an inverse phase precursor. Biophys J. 2009;96(7):2719-2726. doi:10.1016/j.bpj.2008.12.3928

54. Fernandes Patta ACM, Mathews PD, Madrid RRM, Rigoni VLS, Silva ER, Mertins O. Polyionic complexes of chitosan- $\mathrm{N}$-arginine with alginate as $\mathrm{pH}$ responsive and mucoadhesive particles for oral drug delivery applications. Int J Biol Macromol. 2020;148:550-564. doi:10.1016/j.ijbiomac.2020.01.160

55. Mathews PD, Mertins $O$. Dispersion of chitosan in liquid crystalline lamellar phase: Production of biofriendly hydrogel of nano cubic topology. Carbohydr Polym. 2017;157:850-857. doi:https://doi.org/10.1016/j.carbpol.2016.10.071

56. Mathews PD, Fernandes Patta ACM, Gonçalves J V, Gama GDS, Garcia ITS, Mertins O. Targeted Drug Delivery and Treatment of Endoparasites with Biocompatible Particles of pH-Responsive Structure. Biomacromolecules. 2018;19(2):499-510. doi:10.1021/acs.biomac.7b01630

57. Madrid RRM, Mathews PD, Patta ACMF, et al. Safety of oral administration of high doses of ivermectin by means of biocompatible polyelectrolytes formulation. Heliyon. 2021;7(1):e05820. doi:10.1016/j.heliyon.2020.e05820

58. Mathews PD, Patta ACMF, Madrid RRM, Ramirez CAB, Mertins O. Efficient treatment of fish intestinal parasites applying membrane-penetrating oral drug delivery nanobioparticle. ACS Biomat Sci Eng. 2021; under review.

59. Madrid RRM, Mertins O, Tavares-Dias M, Flores-Gonzales, Patta ACMF, Ramirez CAB, Rigori VLS, Mathews PD. High compliance and effective treatment of fish endoparasitic infections with oral drug delivery nanobioparticles: Safety of intestinal tissue and blood parameters. J Fish Dis. 2021. doi: 10.1111/jfd.13501

60. Sohail MF, Rehman M, Sarwar HS, et al. Advancements in the oral delivery of Docetaxel: challenges, current state-of-the-art and future trends. Int J Nanomedicine. 
2018;13:3145-3161. doi:10.2147/IJN.S164518

61. Swarnakar NK, Thanki K, Jain S. Bicontinuous cubic liquid crystalline nanoparticles for oral delivery of Doxorubicin: implications on bioavailability, therapeutic efficacy, and cardiotoxicity. Pharm Res. 2014;31(5):1219-1238. doi:10.1007/s11095-013-1244-8

62. Otte A, Soh B-K, Yoon G, Park K. Liquid crystalline drug delivery vehicles for oral and IV/subcutaneous administration of poorly soluble (and soluble) drugs. Int J Pharm. 2018;539(1-2):175-183. doi:10.1016/j.ijpharm.2018.01.037

63. Nielsen LH, Rades T, Boyd B, Boisen A. Microcontainers as an oral delivery system for spray dried cubosomes containing ovalbumin. Eur J Pharm Biopharm Off J Arbeitsgemeinschaft fur Pharm Verfahrenstechnik eV. 2017;118:13-20. doi:10.1016/j.ejpb.2016.12.008

64. David G, Pérez J. Combined sampler robot and high-performance liquid chromatography: a fully automated system for biological small-angle X-ray scattering experiments at the Synchrotron SOLEIL SWING beamline. I Appl Crystallogr. 2009;42(5):892-900. doi:https://doi.org/10.1107/\$0021889809029288

65. Johnsson M, Barauskas J, Tiberg F. Cubic Phases and Cubic Phase Dispersions in a Phospholipid-Based System. J Am Chem Soc. 2005;127(4):1076-1077. doi:10.1021/ja043331a

66. Murgia S, Falchi AM, Meli V, Schillén K, Lippolis V, Monduzzi M, Rosa A, Schmidt J, Talmon Y, Bizzarri R, Caltagirone C. Cubosome formulations stabilized by a dansylconjugated block copolymer for possible nanomedicine applications. Colloids Surfaces B Biointerfaces. 2015;129:87-94. doi:10.1016/j.colsurfb.2015.03.025

67. Beattie JK, Djerdjev AM. The pristine oil/water interface: surfactant-free hydroxidecharged emulsions. Angew Chem Int Ed Engl. 2004;43(27):3568-3571. doi: 10.1002/anie.200453916

68. Angelova A, Drechsler M, Garamus VM, Angelov A. Liquid crystalline nanostructures as PEGylated reservoirs of omega-3 polyunsaturated fatty acids: Structural insights toward delivery formulations against neurodegenerative disorders. ACS Omega. 2018;3(3):3235-3247. doi: 10.1021/acsomega.7b01935.

69. Simsek-Ege FA, Bond GM, Stringer J. Polyelectrolyte complex formation between alginate and chitosan as a function of pH. J Appl Polym Sci. 2003;88(2):346-351. doi:https://doi.org/10.1002/app.11989

70. Fitch CA, Platzer G, Okon M, Garcia-Moreno BE, McIntosh LP. Arginine: Its pKa value revisited. Protein Sci. 2015;24(5):752-761. doi:10.1002/pro.2647

71. Zhang $X, X i a o ~ Y$, Huang Z, et al. Smart phase transformation system based on lyotropic liquid crystalline@hard capsules for sustained release of hydrophilic and hydrophobic drugs. Drug Deliv. 2020;27(1):449-459. doi:10.1080/10717544.2020.1736210

72. Rossi S, Ferrari F, Bonferoni MC, Caramella C. Characterization of chitosan hydrochloride-mucin interaction by means of viscosimetric and turbidimetric measurements. Eur J Pharm Sci. 2000;10(4):251-257. doi:https://doi.org/10.1016/S0928-0987(00)00065-8

73. Sogias IA, Williams AC, Khutoryanskiy $V$ V. Why is chitosan mucoadhesive? Biomacromolecules. 2008;9(7):1837-1842. doi:10.1021/bm800276d

74. Hayton WL. Rate-limiting barriers to intestinal drug absorption: a review. J Pharmacokinet Biopharm. 1980;8(4):321-334. doi:10.1007/BF01059381 